\title{
New Trends of Emerging Technologies in Digital Pathology
}

\author{
Gloria Bueno $^{\mathrm{a}}$ M. Milagro Fernández-Carrobles ${ }^{\mathrm{a}}$ Oscar Deniz $^{\mathrm{a}}$ \\ Marcial García-Rojo ${ }^{b}$ \\ aVISILAB Group, Escuela Técnica Superior de Ingenieros Industriales, Universidad de Castilla-La Mancha, Ciudad Real, \\ and ${ }^{b}$ Department of Pathology, Hospital de Jerez de la Frontera, Jerez de la Frontera, Spain
}

\section{Key Words}

Digital pathology $\cdot$ Image analysis · Standards .

Virtual microscopy $\cdot$ Whole-slide imaging

\begin{abstract}
The future paradigm of pathology will be digital. Instead of conventional microscopy, a pathologist will perform a diagnosis through interacting with images on computer screens and performing quantitative analysis. The fourth generation of virtual slide telepathology systems, so-called virtual microscopy and whole-slide imaging (WSI), has allowed for the storage and fast dissemination of image data in pathology and other biomedical areas. These novel digital imaging modalities encompass high-resolution scanning of tissue slides and derived technologies, including automatic digitization and computational processing of whole microscopic slides. Moreover, automated image analysis with WSI can extract specific diagnostic features of diseases and quantify individual components of these features to support diagnoses and provide informative clinical measures of disease. Therefore, the challenge is to apply information technology and image analysis methods to exploit the new and emerging digital pathology technologies effectively in order to process and model all the data and information contained in WSI. The fi-
\end{abstract}

nal objective is to support the complex workflow from specimen receipt to anatomic pathology report transmission, that is, to improve diagnosis both in terms of pathologists' efficiency and with new information. This article reviews the main concerns about and novel methods of digital pathology discussed at the latest workshop in the field carried out within the European project AIDPATH (Academia and Industry Collaboration for Digital Pathology).

두 2016 S. Karger AG, Base

\section{Introduction}

In recent years, digital imaging has been applied to many medical fields. Due to technological improvements in hard- and software, digital microscopy became an important diagnostic tool in surgical pathology [1]. Digital microscopy creates the digital version of whole glass slides (whole-slide imaging; WSI), which can be dynamically viewed, navigated and magnified on computer monitors across a computer network. Digital slides can be integrated into existing hospital databases and accessed through the intranet or internet for teaching, primary diagnosis, teleconsultation and quality assurance.

\section{KARGER}

E-Mail karger@karger.com www.karger.com/pat
(C) 2016 S. Karger AG, Basel

$1015-2008 / 16 / 0833-0061 \$ 39.50 / 0$ 
WSI continues to gain attraction among pathologists for diagnostic, educational and research purposes. WSI is already influencing pathology practices, but 'has diagnostic accuracy improved in anatomic pathology?' [2]. Is WSI incorporated in the pathologist workflow? Is WSI used and validated for clinical applications? [3]. 'Are we there yet?' [4]. Problems include limitations in technology and image quality, problems with the scanning of all material and costs [5], in addition to digital slide storage, inability to handle high-throughput routine work, regulatory barriers and pathologists' reluctance [6].

The concept of digital pathology refers to the application of information technology in pathology to support the creation, sharing or exchange of information, including data and images. The final aim is to aid in the complex workflow from specimen receipt to anatomical pathology (AP) report transmission [7]. The main components of digital pathology are: (a) AP information systems and (b) digital image analysis systems, including image acquisition and processing. However, digital pathology is not just connecting scanner devices to AP information systems. It is an integrated effort that requires developing both (a) infrastructure to facilitate the collaboration between different departments and health care systems, and, therefore, use of multimodal and multilevel data generated in AP departments, and (b) image analysis methods to process and model the huge amount of information included in WSIs to provide additional diagnostic information and to assist pathologists during evaluation and diagnosis.

The two key factors that will improve accurate diagnosis are the use of standardized diagnostic language and criteria, as well as the development of digital tools, including classical issues of big data management and image processing [2]. These tools can help to collect and combine all available clinical history data to provide a diagnosis and to connect pathologists for a second opinion.

The development of these tools is only possible through integrated and collaborative projects between the industry and the public sector, including both research institutes and hospitals, focused on developing efficient and innovative products for digital pathology. This is the aim of the European project AIDPATH (Academia and Industry Collaboration for Digital Pathology) [8]. AIDPATH is an IAPP (Industry Academia Partnerships and Pathways) MCA (Marie Curie Action) European Union's 7th Framework Program (FP7) that is going to unfold for 4 years, starting in November 2013. The AIDPATH project is fostering advances in digital pathology through a series of work packages and activities, including work- shops, where the new trends and emerging technologies in digital pathology are reviewed.

This paper summarizes the conclusions of the 2 nd and 3rd AIDPATH workshops. The 2nd workshop, entitled Information Technology in Digital Pathology, was a satellite event of the 'Medical Informatics Europe' Conference conducted by the European Federation for Medical Informatics (EFMI). Therefore, the topics covered different aspects related to the EFMI working groups, such as MIP (Medical Image Processing), IDeS (Information and Decision Support in Biomedical and Health Care) and THI (Translational Health Informatics), all tailored to digital pathology. The 3rd workshop, entitled New Trends in Digital Pathology, was an event within the Joint Meeting of the British Division of the IAP (International Academy of Pathology) and the Pathological Society of Great Britain and Ireland (http://www.path.org.uk/). Most of the references of this paper are part of the research work of the invited speakers to theses workshops (http://aidpath.eu/?page_id=40).

For making medical decisions, health care professionals require that all necessary information is both correct and easily available [9]. Collaboration in digital pathology is supported by standardization efforts towards interoperability and knowledge representation for sharable and computable clinical information. These efforts will be discussed in the following Section: Standards in Digital Pathology.

Digital image analysis is now available in a variety of platforms to improve quantification performance of diagnostic pathology. The advances in diagnosis using WSI processing tools and the emerging research in this area will be the topic of Image Analysis in Digital Pathology. Finally, the conclusions will be drawn.

\section{Standards in Digital Pathology}

Data representation, coding and communication standards in pathology are important concepts that facilitate communication between pathologists and other medical departments. An understanding of the basics of data representation has implications that affect a pathologist's daily practice by optimizing a physician's workflow [10], i.e. the knowledge of which variables must be included in pathology reports and how they are arranged. Moreover, medical coding is required to document pathology services in a way other departments understand.

The use of codes, such as CPT (Current Procedural Terminology) and ICD (International Classification of
Bueno/Fernández-Carrobles/Deniz/ García-Rojo 
Diseases), summarizes many services and diagnoses by means of alphanumeric codes, which are also understood by other actors (e.g. researchers or clinicians, for example). Information systems that follow standards for data representation can transmit and accept these variables more easily. Pathologists' input in the design of electronic medical records, as well as interfaces between these electronic medical records and their relation to AP information systems, is essential to ensure that data are properly represented [11].

A standard is a formal terminology that establishes uniform criteria, methods, processes and practices. Communication standards are crucial to understand and share information. Existing standards range from units, formats, terms to entities. Standards in pathology may be identified with units of measurement (i.e. microns) adopted by the International System of Units (SI) and formats for image representation, such as DICOM (Digital Imaging and Communications in Medicine). A DICOM working group (WG-26) in pathology was created in 2005. The goal of WG-26 is to extend minimal capabilities to describe specimens in DICOM (Supplement 122), to create a mechanism to allow exchange and use of WSI within DICOM (Supplement 145) [12], to include other imaging modalities, such as multispectral images, electron microscopy, flow cytometry and clinical laboratory images, for example, and to facilitate pathology imaging workflow and overall specimen tracking. In other fields, such as radiology, image formats have already been standardized with a DICOM format and are archived with PACS (Picture Archiving and Communication System), but this is not the case in pathology. Currently, no WSI is used in DICOM format.

An example of standards applied to terms is SNOMED CT (Systematized Nomenclature of Medicine Clinical Terms) created by the College of American Pathologists and the National Health Service of the United Kingdom, and maintained by the International Health Terminology Standards Development Organization. Although SNOMED CT codes offer flexibility to provide codes for pathologists, care must be taken to cover the entire histological context. Some studies are concerned that histopathological findings may not be properly represented for patient information exchange and research unless further modifications to SNOMED CT models are developed [13].

Standard entity is Health Level Seven International (HL7), an international organization for hospital information systems accredited by the American National Standards Institute. HL7 allows for messaging protocols

New Trends of Emerging Technologies in Digital Pathology that simplify the interface between health care software applications and vendors. If two different information systems follow HL7, they can provide patient and clinical information, as well as diagnoses between each other without loss or misinterpretation of information.

Some initiatives to promote the adoption of standards in pathology have been done at European level under the COST Action EuroTelepath [14]. The methodology of Integrating the Healthcare Enterprise (IHE) initiative was used in the AP domain [7]. The goal of IHE is to improve the way computer systems share information. The IHE process is based on working groups that include both health care providers and information system vendors in charge of defining domain-specific integration profiles taking into account well-established health care data standards (such as DICOM and HL7) [9]. IHE also stages Connectathons and Interoperability Showcases, in which vendors meet to demonstrate the interoperability of their products. This allows to define new profiles by users and adoption of standards into products. The IHE and content profiles, developed as a result of the AP IHE initiative, support the AP workflow, the AP reporting to public health and the AP structured report with the standardized AP vocabulary called PathLex [15]. Thus, this initiative successfully supports basic image acquisition and reporting processes in AP laboratories and provides a standard solution for sharing structured AP reports in which observations can be explicitly bound to WSI or to regions of interest (ROI) in images [9].

Beside the efforts, there is very little standardization within pathology. Standards in pathology reporting are not widely addressed, except for some standards which emerged for malignant neoplasms [16]. It is usually up to the pathologists' criteria to decide how to report these findings. Moreover, each AP department has their own way of staining, sectioning, imaging, storing and even scoring the slides. Advances in the standardization of digital pathology have been mainly limited by the industry. Another obstacle is that commercial solution providers often create their own proprietary methods, making it impossible to combine technology, equipment or software from different companies [17]. A lack of universal standards is one of the major barriers that have limited the widespread use of digital pathology [6].

An open question arises: Is it time to merge with IHE laboratory domains to better address the new challenges of digital pathology? The risk involved in merging laboratory and AP domains is the possibly decreased visibility of the AP community and less focus on specific AP needs. However, the challenges are both better coverage of the 


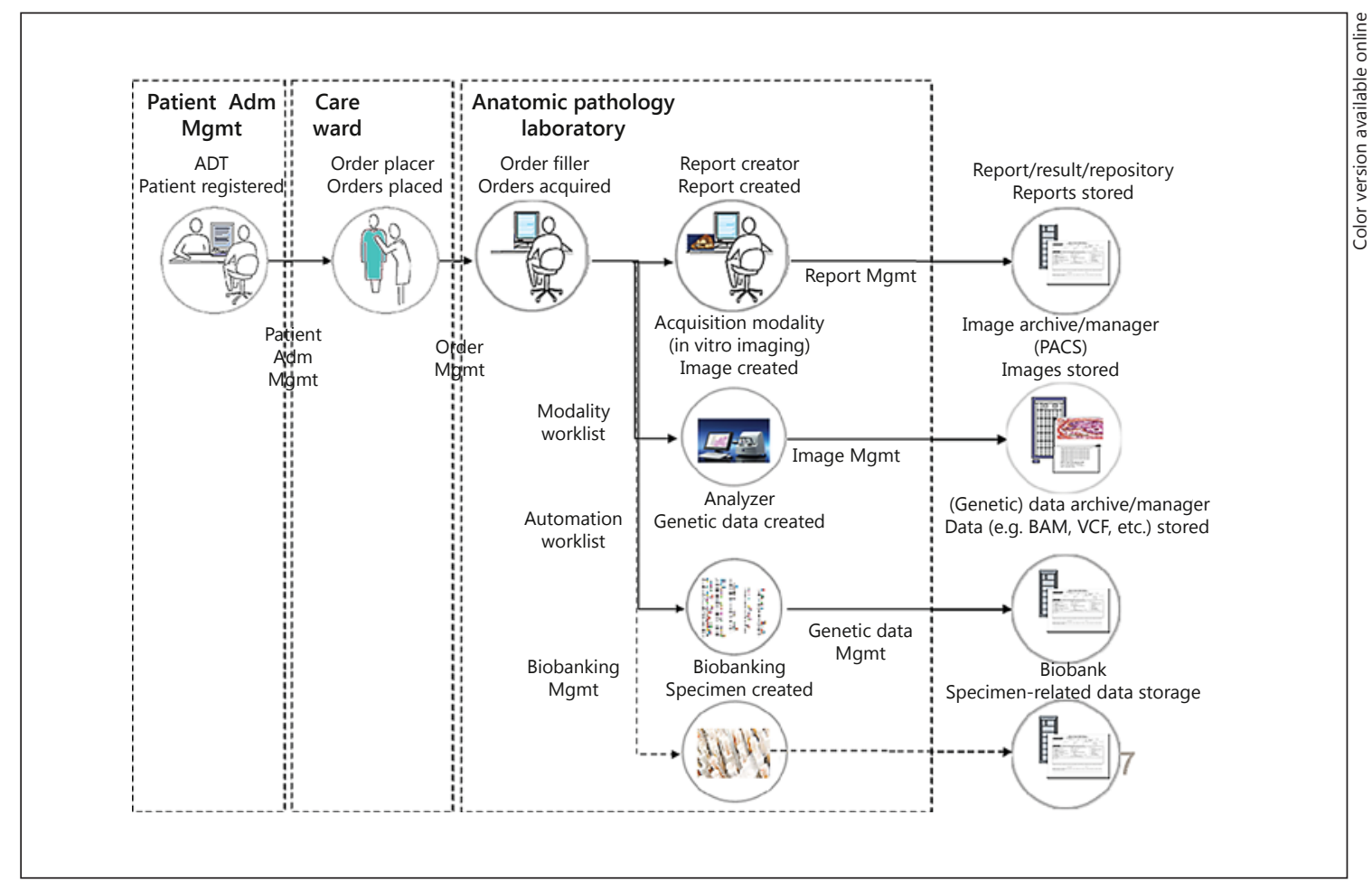

Fig. 1. Extended scope of digital pathology. ADT = Admission (Adm), discharge and transfer; CLM = conventional light microscopy; Mgmt = management.

extended scope of digital pathology and better involvement/adoption by vendors. The coverage of the extended scope of digital pathology can be achieved by means of: (a) new integration profiles for in vitro imaging, to obtain a better definition and management of imaging procedures; (b) new integration profiles for the AP structured report for a better integration of molecular biology and clinical genomics, as well as better alignment to standard terminologies [Logical Observation Identifiers Names and Codes (LOINC), ICD-O, SNOMED CT] and DICOM SR (Supplement 155); (c) use of laboratory integration profiles defined for automation, and (d) improving efficiency in addressing new challenges, e.g. biobanking and NGS (next-generation sequencing).

Figure 1 shows the extended scope of digital pathology where clinical genomics is represented. Knowledge of the human genome is far from complete, but there are already uses for genetic and genomic information in the clinic. Genome sequencing is expected to have the most impact in categorizing patients for appropriate cancer treatment; characterizing and diagnosing genetic diseases, and providing information about genomic alterations in order to match small-molecule drugs to specific disease types. In the near future, researchers expect to gather a broad range of variants that will be clinically useful. Interestingly, the application of genomic medicine may not be limited to typically genetic conditions. Comprehensive profiles of an individual's genetic data combined with common clinical markers are expected to provide new insights into genetic and infectious diseases. This must be taken into account and, therefore, integrated into the extended scope of digital pathology.

\section{Image Analysis in Digital Pathology}

Automatic image analysis has many potential advantages, including reducing interobserver discrepancy, increasing consistency and improving efficiency. Furthermore, the amount of information included in WSI presents tremendous opportunities for developing and evaluating new and more effective treatments that may revolutionize the care of patients with cancers and other diseases.

However, image analysis algorithms in digital pathology are still under research and there are many challeng- 
ing problems and open questions, such as image quality, calibration and optimizing display, development of image processing and machine learning methods for computer-aided diagnostic systems as well as their validation for diagnostic use.

One of the main concerns is if WSI can replace conventional light microscopy to render diagnoses. There are some studies were the use of WSI usually shows good agreement with glass slides [18]. Discrepancies in diagnoses were attributed to image quality, rarely missed tissue on the digital image, inadequate clinical metadata and pathologists' lack of experience using the WSI system [19]. There are no standard guidelines regarding validation of WSI for diagnostic use but just some recent recommendations [19]. Though some researches have compared the quality of images captured with optical microscopes and scanners [20], no metrics are currently applied to WSI to quantify its quality. Is WSI acquired with scanners of equal or better quality than images obtained with a microscope [21]?

WSI quality should be analyzed at different levels, such as focus, color, contrast, resolution, noise and distorted tissue artifacts. All these issues may interfere with image analysis algorithms [22]. Some recent advances have been done for color standardization in WSI by means of using a color calibration slide [23]. A solution to overcome tissue fold artifacts by color enhancement techniques has also been developed by the same authors [24]. Another issue regarding color is the color space used to represent WSI in order to obtain an optimal visualization. There are some recommendations for using a perceptually uniform color space in digital pathology [25], but there is no agreed standard on how color information needs to be visualized for medical applications. This can make quality assurance a challenge. It has been shown that the color space of the display has a significant impact on the perception of clinically relevant areas of digital pathology images [26]. There are some recent studies on the effect of display resolution, not the display of size, on time to diagnosis with WSI [27]. However, the study is limited to 2 monitors, 9 pathologists and 81 slices [28]. Further clinical tests must be done to analyze how the use of different monitors influences diagnosis.

The challenges in digital pathology have led to improvement in image analysis techniques resulting in better opportunities for the pathologist for treatment of benign tissues. The techniques cover all steps of a computer vision system, i.e. preprocessing, segmentation and classification [29, 30]. WSI processing is slowly becoming more relevant for clinical trials, to ensure proper classifi-

New Trends of Emerging Technologies in Digital Pathology cation of cases and to lower diagnosis discrepancy rates. Combining WSI with image analysis tools allows users to leverage technology to perform tasks that were previously too cumbersome or even impossible for humans to undertake manually. Examples include:

- high-throughput morphological analysis of cases to quantitatively and reproducibly measure histological structures such as tumors [31];

- automated grading of tumors to reduce variability encountered with manual grading [32];

- automated selection of desired ROIs, such as hot spot areas with high proliferative activity) [33];

- vessel quantification for angiogenesis research [34], and

- molecular mapping through multistained WSI alignment [35].

Additional WSI-related technologies currently under investigation include automated image analysis tools for computer-assisted diagnosis. Increasingly, the role of (computer-aided diagnostic) algorithms in medical imaging has escalated to a point where algorithms have been developed for disease detection, diagnosis and prediction of prognosis that complements the opinion of the pathologist. A good review on recent developments in computer-aided diagnostic systems for digitized histopathology can be found in the literature [36].

The value of pattern recognition in histopathology can be seen for different applications. Among others, we can identify the following:

1 it is useful to find patterns in artifacts; hopefully there are consistent patterns of 'consistent artifacts' to enable the analysis of changes in relation to normal tissue changes/tumor differentiation and relationships to drug administration;

2 it provides practical, more robust solution for ROI identification;

3 it obviates bias;

4 it is likely to identify stochastic artifacts in tissue sections, and

5 the potential to use misclassification rate as a marker of response.

For example, in many cases, tissue phenotypes are scored from 1 to 4 , but the truth is that biological heterogeneity is more complex than that. The good news is that image analysis software solutions, including pattern recognition, can provide a quantitative readout capturing the heterogeneity in the tissue. Using software to identify individual objects in a tissue sample and evaluate those objects in relation to the rest of the tissue, you will have a better read on the heterogeneity of your samples and pos- 
sibly a deeper understanding of disease states and prognosis.

Some considerations should be directed to some existing market applications using pattern recognition [37]. A recent review on 5 commercial systems, Aperio/Genie (developed under the name Genie Pro at Los Alamos National Laboratories); Definiens Tissue Studio; TissuemorhDP (Visiopharm); Halo (IndicaLabs) and iPerkinElmer, reveals the following:

1 Methods are sensitive to variations in sample preparation processes, which influence both spectral and texture-based classifiers. The classification methods used are quite simple.

2 They provide classification translation (dedicated application may not translate to other 'like' studies). Therefore, complex tumor/tissue heterogeneity may not be accounted for.

3 They have misclassifications and lack of robustness due to several aspects such as insufficient optimization. Moreover, too many classes increase computational requirements and too few classes provide noisy data.

4 They are specific to object orientation (they may require associated image registration to eliminate the effect of orientation, e.g. polarized cells).

Novel pattern recognition and classification methods, such as deep structured learning or hierarchical learning, as well as ensemble learning and adaptive boosting, should be applied to digital pathology. Moreover, for most of the classification techniques, a good set of features describing the ROIs, i.e. the tissue components and tumor patterns, must be defined.

\section{New Techniques}

Multiplexing is the localization of multiple protein and similar species antibodies in a single section from the same sample. Multiplexing assists in the localization of proteins of interest and concurrent association with cell compartments or cell types. Simultaneously, several aspects of a cell can be measured. Novel works in digital pathology are emerging by using new methods for multiplexing using new protein expression methods and automated multispectral slide imaging techniques. Immunohistochemistry (IHC) methods and multispectral analysis with the image processing and pattern recognition techniques mentioned above are the focus of novel oncological assays. Expression profiles of each marker can be evaluated alone in a singleplex assay, or together within a multiplex assay, in order to verify the utility of this novel multiplex IHC. Multiplexing uses primarily fluorescence with either standard fluorochromes or quantum dots (qdots). In current practice, multiplexed stained samples can be difficult to interpret, since, due to fluorescence, multiple targets can blend together and tissue autofluorescence can appear. Those are sources of visual perception errors. These errors can be addressed by means of multispectral unmixing.

One novel system is the toponome imaging system, i.e. an automated molecular fluorescence tagging and imaging system that yields subcellular colocalization of more than 100 proteins through sequential episodes of tag binding/bleaching to an intact cell or tissue section [38]. Therefore, this novel technology holds promise for developing a new generation of multiplex biomarkers. The integral role of the microenvironment in malignant progression and the recently appreciated heterogeneity of cancer cells stress the importance of characterizing complex molecular phenotypes and the large protein network structures of single cells within their anatomical context. However, the technology requires optimization to improve the reproducibility and accuracy of image analysis, as well as further validation of findings using complementary techniques [38].

Multispectral analysis has broad potential for designing combinatorial therapeutic approaches by revealing coexpression, pathway configurations and spatial relationships among cell types in oncology [39]. Some studies use multispectral analysis for quantitative in situ immunophenotyping in immuno-oncology research [40,41]. Currently, despite advances in technology, the majority of studies in histopathology utilize manual, qualitative analysis of single markers to denote the phenotype. This process is highly subjective with intra-/interobserver variation. On the other hand, multiple marker expression delineates phenotypes - as currently determined using other methods such as flow cytometry - but this has been difficult to achieve using histology-based techniques.

In the past, qdots have been applied to multispectral analysis for multiplex IHC and in situ hybridization [42]. qdots are photostable semiconductor nanocrystals possessing wide excitation spectra and narrow, symmetrical emission spectra. qdots combined with multispectral imaging have enabled to quantitate and determine colocalization of gene expression in clinical tissue. However, for more complex assays, especially using IHC, this methodology has some difficulties, i.e. antibody cross reactivity and the need for individual antibodies to different species, as well as the need for an ever more complex labora-
Bueno/Fernández-Carrobles/Deniz/ García-Rojo 
Fig. 2. Future perspective of digital pathology imaging from inspection in the bright field over the right treatment to the right patient. FISH $=$ Fluorescence in situ hybridization; ISH = in situ hybridization.

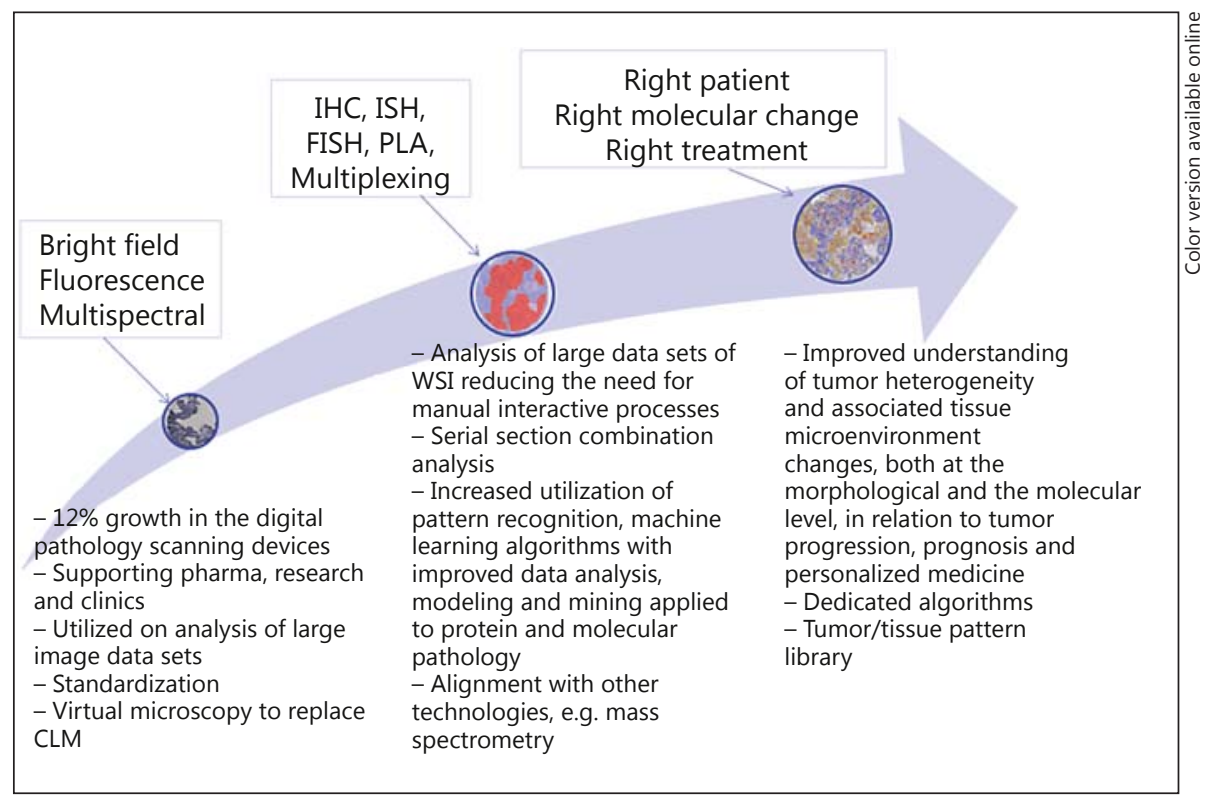

tory workup. In realistic terms, this means going beyond three stains becomes very cumbersome.

Tyramide signal amplifications (TSA) were launched in 2014 as part of the Opal multiplex IHC [43]. TSA reagents address the problems encountered with qdots. They work very much similar to a standard IHC protocol, using the horseradish peroxidase enzyme for protein detection. Moreover, the method enables the deposition of TSA fluors close to the antigen of interest. The nature of the protocol allows for a simplified multiplexing strategy, where primary antibodies can be chosen based on performance and not on species.

In multispectral imaging analysis, the raw image cube, which comprises up to 60 images acquired at regular wavelength intervals, is first taken. Then, previously determined spectral profiles associated with each individual fluorophore are used to spectrally deconvolute the original image into several component images associated with each individual fluorophore. Taking multispectral imaging a step further, we can use image processing methods to segment cells based upon a nuclear stain. Subsequently, it is possible to determine within a specific cell area the colocalization of spectrally deconvoluted stains, using staining thresholds, in order to determine positivity for one or more markers per cell. The added advantage of using image processing is that it is possible to acquire $\mathrm{x}-/ \mathrm{y}$ coordinates of the cells alongside immunophenotypic characteristics. Moreover, further analysis can be done to analyze the texture of pattern biomarkers as well as the interaction distributions of different cell expressions, i.e. cells in close proximity may be interacting and therefore paired-cell/protein interactions (number and distance) may be quantified. The co-occurrence matrix of these interactions can be represented in a heat map format, as PLA (proximity ligation assay), which may be useful for examining invasion and progression of tumors.

Figure 2 shows a scheme of the future perspective of digital pathology imaging taking into account the new trends on the above-described emerging technologies in digital pathology [37].

\section{Conclusions}

Digital pathology and WSI technology have the potential to improve diagnostic accuracy, increase workflow efficiency, balance workloads and better integrate images in information systems. The amount of information that can be extracted from a tissue sample using complex, automated and miniaturized devices will continue to increase thanks to sophisticated computer-based algorithms, which will assist in the integration of all information.

The two main aspects that will help to foster the use of digital pathology in clinical routine are the use of standards and the development and validation of image analysis tools. 
Standardization is needed for pre-imaging steps (e.g. consistent staining and optimal slide preparation without artifacts), image acquisition (e.g. optimal resolution), processes after imaging (e.g. color calibration) and sharing/transmission of digital images (e.g. interoperable file formats).

Furthermore, as more image analysis algorithms and computer-assisted diagnosis tools get developed and validated for clinical use, they will empower pathologists to become more efficient, precise and reproducible at quantifying prognostic biomarkers. Moreover, laboratory and tissue-based diagnostics will increase their capability to provide a safe guide to therapy. Enhanced imaging capabilities will allow groups of pathologists to share information on tissue-based diagnostics. Pathologists sharing knowledge of histopathology, disease-related molecular processes and laboratory diagnostics will be the integra- tors of information related to the molecular, biochemical and cellular processes underlying the patient's disease, complications and symptoms.

Finally, we believe that all of these challenges are affordable and, therefore, digital pathology will continue to transform the practice of pathology, which will provide opportunities for developing and evaluating new and more effective treatments that will benefit the patients.

\section{Acknowledgments}

The authors acknowledge financial support from the European Union's FP7 (MCA) through AIDPATH project contract 6154. The authors would like to thank all research fellows from the AIDPATH consortium that have participated and made the success of all AIDPATH events possible (http://aidpath.eu/).

\section{References}

1 García-Rojo M, Blobel B, Laurinavicius A: Perspectives on Digital Pathology. Amsterdam, IOS, 2012.

$\checkmark 2$ Nakhleh RE: Has diagnostic (analytic) accuracy improved in anatomic pathology? Are we better today than we were 20 years ago? Arch Pathol Lab Med 2015;139:716-718.

>3 Thrall MJ, Wimmer JL, Schwartz MR: Validation of multiple whole slide imaging scanners based on the guideline from the College of American Pathologists Pathology and Laboratory Quality Center. Arch Pathol Lab Med 2015;139:656-664.

4 Hewitt SM: Digital pathology - are we there yet? 8th Congress of the Pathological Society of Great Britain and Ireland, Dublin, 2015. http://www.pathsoc.org/files/meetings/Dublin2015Presentations/Digital\%20Path\%20 -\%201\%20Hewitt.pdf.

5 Ho J, Ahlers SM, Stratman C, et al: Can digital pathology result in cost savings? A financial projection for digital pathology implementation at a large integrated health care organization. J Pathol Inform 2014:5:33.

$\checkmark 6$ Farahani N, Pantanowitz L: Overview of telepathology. Surg Pathol Clin 2015;8:223231.

7 Daniel C, García-Rojo M, Klossa J, Della Mea V, Booker D, Beckwith BA, et al: Standardizing the use of whole slide images in digital pathology. Comput Med Imaging Graph 2011; 35:496-505.

8 AIDPATH: Academia and Industry Collaboration for Digital Pathology. http://aidpath. $\mathrm{eu} /$.

-9 Daniel C, Booker D, Beckwith B, Della Mea V, García-Rojo M, Havener L, Kennedy M, Klos- sa J, Laurinavicius A, et al: Standards and specifications in pathology: image management, report management and terminology. Stud Health Technol Inform 2012;179:105122.

-10 Amin M, Rajiv D: Data representation, coding, and communication standards. Surg Pathol 2015;8:109-121.

11 Hernandez JS, Allen TC: Transformation of pathologists responding in a volatile, uncertain, complex, and ambiguous environment. Arch Pathol Lab Med 2013;137:603-605.

12 Singh R, Chubb L, Pantanowitz L, et al: Standardization in digital pathology: supplement 145 of the DICOM standards. J Pathol Inform 2011;2:23.

113 Campbell WS, Campbell JR, West WW, et al: Semantic analysis of SNOMED CT for a postcoordinated database of histopathology findings. J Am Med Inform Assoc 2014;21:885892.

14 García-Rojo M, Morillo-Castro A, Gonçalves L: COST Action 'EuroTelepath': digital pathology integration in electronic health record, including primary care centres. Diagn Pathol 2011;6(suppl 1):S6.

15 IHE Profiles. http://wiki.ihe.net/index.php? title=Profiles\#IHE_Anatomic_Pathology_ Profiles.

16 Goldsmith JD, Siegal GP, Suster S, et al: Reporting guidelines for clinical pathology reports in surgical pathology. Arch Pathol Lab Med 2008;132:1608-1616.

17 Farahani N, Parwani AW, Pantanowitz L: Whole slide imaging in pathology: advantages, limitations, and emerging perspectives. Pathol Lab Med Int 2015;7:23-33.
18 Bauer T, Slaw RJ: Validating whole-slide imaging for consultation diagnoses in surgical pathology. Arch Pathol Lab Med 2014;138: 1459-1465.

19 Pantanowitz L, Sinard J, Henricks WH, Fatheree LA, Carter AB, Contis L, Beckwith BA, Evans AJ, Otis CN, Lal A, Parwani AV: Validating whole slide imaging for diagnostic purposes in pathology: guideline from the College of American Pathologists Pathology and Laboratory Quality Center. Arch Pathol Lab Med 2013;137:1710-1722.

20 Redondo R, Bueno G, Cristóbal G, Vidal J, Déniz O, García-Rojo M: Quality evaluation of microscopy and scanned histological images for diagnostic purposes. Micron 2012;43: 334-343.

21 Yagi Y, Pantanowitz L: Comment on 'Quality evaluation of microscopy and scanned histological images for diagnostic purposes': are scanners better than microscopes? J Pathol Inform 2012;3:14.

22 Yagi Y, Gilbertson J: A relationship between slide quality and image quality in whole slide imaging. Diagn Pathol 2008;3:S12.

23 Bautista PA, Hashimoto N, Yagi Y: Color standardization in whole slide imaging using a color calibration slide. J Pathol Inform 2014;5:4.

24 Bautista PA, Yagi Y: Improving the visualization and detection of tissue folds in whole slide images through color enhancement. J Pathol Inform 2010;1:25.

25 Avanakia A, Espiga K, Kimpe T, Xthonaa A, Marchessoux C, Rostang J, Piepers B: Perceptual uniformity of commonly used color spaces. Medical Imaging 2014: Digital Pathology. Proc SPIE 2014;9041:90410V. 
26 Kimpe T, Rostang J, Avanaki A, et al: Does the choice of display system influence perception and visibility of clinically relevant features in digital pathology images? Medical Imaging 2014: Digital Pathology. Proc SPIE 2014;9041: 904109.

-27 Randell R, Ambepitiya T, Mello-Thoms C, Ruddle RA, Brettle D, Thomas RG, Treanor D: Effect of display resolution on time to diagnosis with virtual pathology slides in a systematic search task. J Digit Imaging 2015;28: 68-76.

28 García-Rojo M, Bueno G: Analysis of the impact of high-resolution monitors in digital pathology. J Pathol Inform 2015;6:57.

-29 Gurcan MN, Can A, Boucheron L, Madabhushi A, Rajpoot N, Yener B: Histopathological image analysis: a review. IEEE Rev Biomed Eng 2009;2:147-171.

-30 Bhattacharjee S, Mukherjee J, Nag S, Maitra IK, Bandyopadhyay SK: Review on histopathological slide analysis using digital microscopy. Int J Adv Sci Technol 2014;62:65-96.

- 31 Kong J, Cooper LA, Wang F, Gao J, et al: Machine-based morphologic analysis of glioblastoma using whole-slide pathology images uncovers clinically relevant molecular correlates. PLoS One 2013;8:e81049.

32 Yeh FC, Parwani AV, Pantanowitz L, Ho C: Automated grading of renal cell carcinoma using whole slide imaging. J Pathol Inform 2014;5:23.

$33 \mathrm{Lu} \mathrm{H}$, Papathomas TG, Zessen D, et al: Automated selection of hotspots (ASH): enhanced automated segmentation and adaptive step finding for Ki67 hotspot detection in adrenal cortical cancer. Diagn Pathol 2014;9:216.

34 Fernández-Carrobles MM, Tadeo I, Bueno G, Noguera R, Déniz O, Salido J, García-Rojo M: TMA vessel segmentation based on color and morphological features: application to angiogenesis research. ScientificWorldJournal 2013;2013:263190.

35 Deniz O, Toomey D, Conway C, Bueno G: Multi-stained whole slide image alignment in digital pathology. Medical Imaging 2015: Digital Pathology. Proc SPIE 2015;9420: 94200Z.

36 Fernández-Carrobles MM, Bueno G, Déniz O, Salido J, García-Rojo M, González-López $\mathrm{L}$ : Influence of texture and colour in breast TMA classification. PLoS One 2015; 10:e0141556.

37 Bigley A: Application of pattern recognition software on preclinical and safety studies. 8th Congress of the Pathological Society of Great Britain and Ireland, Dublin, 2015, AIDPATH Workhop.

38 Evans RG, Naidu B, Rajpoot MM, Epstein D, Khan M: Toponome imaging system: multi- plex biomarkers in oncology. Trends $\mathrm{Mol}$ Med 2012;18:723-731.

39 Stack EC, Wang C, Roman KA, Hoyt CC: Multiplexed immunohistochemistry, imaging, and quantitation: a review, with an assessment of Tyramide signal amplification, multispectral imaging and multiplex analysis. Methods 2014;70:46-58

40 Slater C: Multispectral imaging and image analysis software can accurately quantify immunophenotypically distinct tumour infiltrating lymphocytes (TIL) using chromogenic multiplexed immunohistochemistry (IHC) in lymphoid tissue microarrays (TMAs). 26th European Congress of Pathology, London, August 30 to September 3, 2014.

41 Byers R, Hornick JL, Tholouli E, Kutok J, Rodig JS: Detection of IDH1 R132H mutation in acute myeloid leukemia by mutation-specific immunohistochemistry. Appl Immunohistochem Mol Morphol 2012;20:37-40.

42 Byers RJ, Hitchman ER: Quantum dots brighten biological imaging. Prog Histochem Cytochem 2011;45:201-237.

43 Slater C: Investigating the prognostic significance of tumour infiltrating lymphocytes (TILs) in post-transplant lymphoproliferative disorder (PTLD): a novel approach. 26th European Congress of Pathology, London, August 30 to September 3, 2014. 